\title{
Does Extended Lymphadenectomy Help in Pancreatic Cancer?
}

\author{
Hassan Aziz, MD ${ }^{1}$, Jordan M. Cloyd, $\mathrm{MD}^{2}$, Gaya Spolverato, $\mathrm{MD}^{3}$, and Timothy M. Pawlik, MD, MPH, MTS, \\ $\mathbf{P h D}^{2}$
}

${ }^{1}$ Department of Surgery, Tufts University School of Medicine, Boston, MA; ${ }^{2}$ Department of Surgery, The Ohio State University Wexner Medical Center, Columbus, $\mathrm{OH} ;{ }^{3}$ Department of Surgical Oncological and Gastrointestinal Sciences, University of Padua, Padua, Italy

Pancreatic ductal adenocarcinoma (PDAC) is a lethal disease with a median 5 year survival of $2-9 \% .{ }^{1}$ Routine lymphadenectomy is considered an important component of curative-intent pancreatectomy. ${ }^{1,2}$ The American College of Surgeons Operative Standards for Cancer Surgery recommends routine dissection of the nodal basins along the "common bile duct, common hepatic artery, portal vein, posterior and anterior pancreaticoduodenal arcades, superior mesenteric vein, and right lateral wall of the superior mesenteric artery" during pancreatoduodenectomy and "along the splenic artery, at the supra- and infrapancreatic border, and at the splenic hilum" for distal pancreatectomy. ${ }^{2}$ Resection of additional lymph nodes beyond these basins is typically referred to as extended lymphadenectomy.

The primary purpose of lymphadenectomy for cancer is proper staging. However, there has been a long-standing debate about the contribution of lymphadenectomy extent to improve survival or at least locoregional cancer control. In fact, repeated attempts to broaden the extent of lymphadenectomy for PDAC have failed to demonstrate a survival benefit yet have been associated with worsened perioperative and quality of life outcomes (Table 1). ${ }^{3,4}$ The argument against extended lymph node resection stems from the argument that only complete resection of all microscopic disease (including systemic, local, and regional) will lead to acceptable long-term outcomes for patients

(C) Society of Surgical Oncology 2022

First Received: 30 November 2021

Accepted: 16 January 2022;

Published Online: 4 February 2022

T. M. Pawlik, MD, MPH, MTS, PhD

e-mail: Tim.Pawlik@osumc.edu with pancreatic cancer, and that a only a small minority of patients will recur with isolated unresected second echelon metastatic lymph nodes. ${ }^{5}$ Indeed, extended lymphadenectomy is likely only to help if the following criteria have been met: (1) R0 resection has been achieved, (2) there is no macro- or micrometastatic disease (presumed present in the majority of patients at diagnosis), and (3) there are second echelon metastatic lymph nodes (removing negative lymph nodes in secondary basins would not improve disease control). Therefore, while advances in surgical techniques to facilitate receipt of R0 resection for more patients are warranted, extended lymphadenectomy is likely to provide a survival benefit to only a very few patients, as had been demonstrated previously. ${ }^{6}$

Nevertheless, two recent retrospective studies have again attempted to evaluate the potential role of extended lymphadenectomy during pancreatectomy for PDAC. In the recent study by Hirono et al. that included 167 patients who underwent upfront pancreaticoduodenectomy (PD) for resectable PDAC, the authors concluded that complete circumferential lymphadenectomy around the superior mesenteric artery (SMA) may contribute to local control. ${ }^{5}$ Specifically, $360^{\circ}$ circumferential lymphadenectomy around the SMA was performed in 75 patients, and right semi-circumferential lymphadenectomy around the SMA in the remaining 92 patients. Of note, only $60 \%$ of patients had an R0 resection, and $63 \%$ had metastatic lymph nodes. Furthermore, $70 \%$ of local recurrences occurred around the SMA. The authors noted that right-half only lymphadenectomy around the SMA was associated with a higher risk of locoregional recurrence, while complete circumferential lymphadenectomy demonstrated a lower risk of locoregional recurrence on propensity score analysis. These data should be interpreted with caution, however. The authors utilized a Cox proportional hazards 
TABLE 1 Selected studies examining the impact of lymph node retrieval on long-term outcomes following pancreatic resection of adenocarcinoma

\begin{tabular}{|c|c|c|c|c|c|}
\hline Trial & Year & Group & Lymph node retrieval & Overall survival & Mortality \\
\hline \multirow[t]{2}{*}{ Pedrazzoli } & 1991-1994 & 40 standard & Mean: 13.3 & All patients: 2 years: $22.2 \%$ & $5 \%$ \\
\hline & & 41 extended & & 3 years: $8.6 \% ; 4$ years: $7.4 \%$ & $4.9 \%$ \\
\hline \multirow[t]{2}{*}{ Yeo } & 1996-2001 & 146 standard & Mean: 17; median: 16 & PDAC only: 3 years: $36 \% ; 5$ years: $10 \%$ & $4 \%$ \\
\hline & & $\begin{array}{l}148 \\
\quad \text { extended }\end{array}$ & $\begin{array}{l}\text { Mean: } 28.5 \text {; median: } 26 \\
\quad p<0.001\end{array}$ & $\begin{array}{l}\text { PDAC only: } 3 \text { years: } 38 \% ; 5 \text { years: } 25 \%, p= \\
0.57\end{array}$ & $2 \%$ \\
\hline \multirow[t]{2}{*}{ Farnell } & $1997-2003$ & 40 standard & Mean: 15 ; median: 15 & 3 years: $41 \%$; 5 years: $16.4 \%$ & $0 \%$ \\
\hline & & 39 extended & Mean: 34; median: $34, p<0.001$ & 3 years: $25 \% ; 5$ years: $16.5 \%, p=0.32$ & $3 \%$ \\
\hline \multirow[t]{2}{*}{ Nimura } & $2000-2003$ & 56 standard & Mean: 13.3 & 5-year OS: $15.7 \%$ & NA \\
\hline & & 56 extended & Mean: $40.1, p<0.0001$ & 5-year OS: $6.0 \%$ & NA \\
\hline \multirow[t]{2}{*}{ Jang } & $2006-2009$ & 101 standard & $17.3 \pm 10.6$ & 2-year OS: $44.5 \%$ & 0 \\
\hline & & 99 extended & $33.7 \pm 15.1, p<0.001$ & 2-year OS: $35.7 \%$ & $2.3 \% \mathrm{NS}$ \\
\hline \multirow[t]{2}{*}{ Jang } & 2006-2009 & 83 standard & $17.3 \pm 10.5$ & 5-year OS: 18.4 & N/A \\
\hline & & 86 extended & $33.7 \pm 15.1, p<0.001$ & 5-year OS: $14.4 \%, p=0.388$ & N/A \\
\hline \multirow[t]{2}{*}{ Ignjatovic } & $2007-2010$ & 30 standard & $18.5 \pm 5$ & 5-year OS: $6.9 \%$ & $3.30 \%$ \\
\hline & & 30 standard & $24.1 \pm 6.5, p<0.01$ & 5-year OS: $7.1 \%, p>0.05$ & $\begin{array}{l}6.7 \%, p> \\
0.05\end{array}$ \\
\hline
\end{tabular}

regression model, which is typically used to investigate the effect that a given variable has on the time to a specified event. Given the relatively short median follow-up time of 29.3 months, circumferential lymphadenectomy may not have been associated with an absolute reduced risk of local recurrence, but rather just a delay in locoregional regional recurrence. In addition, propensity score matching is generally better suited for the analysis of datasets with larger sample sizes than in the Hirono et al. study. In addition, propensity score matching is susceptible to residual bias as nonmeasured confounders are unaccounted for and may result in residual selection bias-important factors given the reported $p$-value and large 95\% confidence intervals.

In a separate single-center retrospective study by Kobayashi, 83 patients with T3 PDAC were categorized into two groups based on the extent of lymphadenectomy. Patients who underwent complete lymphadenectomy around the SMA had a longer median survival time (40.3 vs. 22.6 months), as well as a higher 5-year survival ( $40.3 \%$ vs. $5.9 \%$ ) than the conventional group. Recurrence around the SMA was less frequent in the artery-first approach group than in the conventional group $(22.2 \%$ vs. $44.2 \%){ }^{6}$ While 88 patients may be a reasonable sample size, the number of "events" (i.e., recurrence around the SMA) was very small $(<20$ in each group). While the difference was "statistically significant" with a $p$-value of 0.041 , the comparison was assessed using only a simple Chi square test, with no provision of the effect estimate or the $95 \%$ CI (which undoubtedly would have been very large). In addition, the two groups were from different time periods, with the conventional lymphadenectomy group having been operated on over 10 years ago. In essence, the authors were reporting on 88 patients over a 17 year period (or about 5 per year, which is very low volume). Furthermore, no patient was treated with preoperative chemotherapy, which is different than the real-world clinical practice in the United States where the vast majority of patients-even those with clearly resectable disease-are now treated with chemotherapy first. While recurrence around the SMA was associated with worse overall survival, it is hard to conclude from these retrospective data that this pattern of recurrence "caused" worse survival rather than it just being a harbinger of worse biology and, therefore, a worse prognosis.

More than 20 years ago, Dr. Blake Cady proclaimed that "biology is king, selection of cases is queen, and the technical details of surgical procedures are princes and princesses of the realm who frequently try to overthrow the powerful forces of the king and queen, usually to no long term avail, although with some apparent temporary victories." 3 While surgical technique and expertise remain important, 'more' surgery does not always lead to better patient outcomes. The goals of surgical resection, as for almost all gastrointestinal cancers, is to achieve a marginnegative resection and perform appropriate regional lymphadenectomy for staging purposes, while minimizing postoperative morbidity in order to facilitate receipt of necessary adjuvant therapies. At this point, the data would suggest that more extensive surgery beyond these goals likely only contributes to worsened morbidity and quality of life. ${ }^{7}$ Careful patient selection, application of standardsbased oncologic principles at the time of surgery, and 
collaboration with an experienced multidisciplinary team that effectively delivers multimodality therapy will ensure the best patient outcomes.

\section{DECLARATIONS}

\section{CONFLICT OF INTEREST None.}

\section{REFERENCES}

1. Morales-Oyarvide V, Rubinson DA, Dunne RF, Kozak MM, Bui JL, Yuan C, Qian ZR, Babic A, Da Silva A, Nowak JA, et al. Lymph node metastases in resected pancreatic ductal adenocarcinoma: predictors of disease recurrence and survival. Br J Cancer. 2017;117(12):1874-82.

2. Operative Standards for Cancer Surgery. American college of surgeons, https://www.facs.org/quality-programs/cancer/acs-crp/o scs.

3. Dillhoff M, Pawlik TM. Role of node dissection in pancreatic tumor resection. Ann Surg Oncol. 2021;28(4):2374-81. https://doi. org/10.1245/s10434-020-09394-6.

4. Pawlik TM, Abdalla EK, Barnett CC, et al. Feasibility of a randomized trial of extended lymphadenectomy for pancreatic cancer. Arch Surg. 2005;140(6):584-91. https://doi.org/10.1001/a rchsurg.140.6.584.

5. Hirono S, Kawai M, Okada KI, et al. Complete circumferential lymphadenectomy around the superior mesenteric artery with preservation of nerve plexus reduces locoregional recurrence after pancreatoduodenectomy for resectable pancreatic ductal adenocarcinoma. Eur J Surg Oncol. 2021;47(10):2586-94. https://doi. org/10.1016/j.ejso.2021.06.005.

6. Kobayashi S, Otsubo T, Nakano H, Koizumi S, Nakahara K. Complete lymphadenectomy around the entire superior mesenteric artery improves survival in artery-first approach pancreatoduodenectomy for T3 pancreatic ductal adenocarcinoma. World $J$ Surg. 2021;45(3):857-64. https://doi.org/10.1007/s00268-020-058 56-w.

7. Cloyd JM, Heh V, Pawlik TM, et al. Neoadjuvant therapy for resectable and borderline resectable pancreatic cancer: a metaanalysis of randomized controlled trials. $J$ Clin Med. 2020;9(4):1129. https://doi.org/10.3390/jcm9041129.

Publisher's Note Springer Nature remains neutral with regard to jurisdictional claims in published maps and institutional affiliations. 